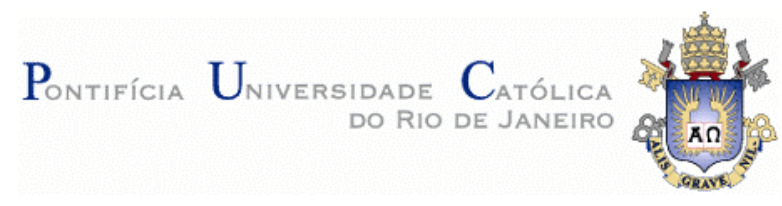

Thiago Barra Vidal de Oliveira

Avaliação metrológica do desempenho do medidor tipo v-cone para medição de vazão de gás úmido

Dissertação apresentada como requisito parcial para obtenção do título de Mestre pelo Programa de PósGraduação em Metrologia da PUC-Rio. Área de Concentração: Metrologia para Qualidade e Inovação.

Orientador: Prof. Alcir de Faro Orlando

Rio de Janeiro

Setembro de 2010 
Thiago Barra Vidal de Oliveira

\section{Avaliação metrológica do desempenho do medidor tipo v-cone para medição de vazão de gás úmido}

Dissertação apresentada como requisito parcial para obtenção do grau de Mestre pelo Programa de PósGraduação em Metrologia do Centro Técnico Científico da PUC-Rio. Aprovada pela Comissão Examinadora e homologada pela Coordenação Setorial de PósGraduação, formalizado pelas respectivas assinaturas.

Prof. Dr. Alcir de Faro Orlando

Orientador

Programa de Pós-Graduação em Metrologia (PósMQI)

Departamento de Engenharia Mecânica Pontifícia Universidade Católica do Rio de Janeiro (PUC-Rio)

Prof. Dr. Marcos Sebastião de Paula Gomes

Departamento de Engenharia Mecânica Pontifícia Universidade Católica do Rio de Janeiro (PUC-Rio)

Prof. Dr. Luis Fernando Alzuguir Azevedo

Departamento de Engenharia Mecânica Pontifícia Universidade Católica do Rio de Janeiro (PUC-Rio)

Dra. Maria Helena Farias Instituto Nacional de Metrologia, Normalização e Qualidade Industrial INMETRO

Prof. José Eugênio Leal Coordenador Setorial de Pós-Graduação do Centro Técnico Científico (PUC-Rio) 
Todos os direitos reservados. É proibida a reprodução total ou parcial do trabalho sem autorização da universidade, do autor e do orientador.

Thiago Barra Vidal de Oliveira

Graduou-se em Engenharia de Controle e Automação pela Universidade Federal de Minas Gerais - UFMG - em 2003. Cursou Especialização em Engenharia de Petróleo pela Universidade Petrobras, com conclusão em 2005. Engenheiro de Automação da empresa ATAN Sistemas de Automação e Otimização de 2003 a 2004. Engenheiro de Petróleo da Petrobras desde 2004, atuando no segmento de medição de vazão de petróleo e gás natural nas Unidades de Exploração e Produção (E\&P).

Ficha Catalográfica

\section{Oliveira, Thiago Barra Vidal de}

Avaliação metrológica do desempenho do medidor tipo vcone para medição de vazão de gás úmido / Thiago Barra Vidal de Oliveira ; orientador: Prof. Dr. Alcir de Faro Orlando - 2010.

166 f. : il. (color.) ; $30 \mathrm{~cm}$

Dissertação (Mestrado em Metrologia) - Pontifícia Universidade Católica do Rio de Janeiro, Centro Técnico Científico, Rio de Janeiro, 2010.

Inclui bibliografia.

1. Metrologia - Teses. 2. Medição de vazão. 3. Gás úmido. 4. Medidor tipo v-cone. I. Orlando, Alcir de Faro. II. Pontifícia Universidade Católica do Rio de Janeiro. Programa de PósGraduação em Metrologia para Qualidade e Inovação. III. Título. 


\section{Agradecimentos}

A Deus, pela realização deste projeto.

Ao Professor Alcir, pela orientação prestada em cada etapa do trabalho.

À Petrobras, pelo incentivo ao desenvolvimento profissional.

Ao Grupo de Medição do E\&P-ENGP e demais colegas da Petrobras, pelas contribuições realizadas para o desenvolvimento deste trabalho.

Aos meus pais, pelo esforço dedicado para me proporcionar uma educação de qualidade.

À minha namorada, pela paciência e companheirismo.

Aos meus amigos e demais familiares, pelo apoio incondicional em todos os momentos.

Aos professores, funcionários e colegas da PUC-Rio, que estiveram presentes ao longo desta caminhada.

Aos professores da banca examinadora, por dedicarem seu tempo à avaliação do trabalho, contribuindo para a sua melhoria. 


\section{Resumo}

Barra Vidal de Oliveira, Thiago; Orlando, Alcir de Faro. Avaliação metrológica do desempenho do medidor tipo v-cone para medição de vazão de gás úmido. Rio de Janeiro, 2010. 166p. Dissertação de Mestrado - Programa de Pós-Graduação em Metrologia. Departamento de Metrologia para Qualidade Industrial, Pontifícia Universidade Católica do Rio de Janeiro.

O objetivo deste trabalho é avaliar o desempenho metrológico e o potencial de aplicação do medidor tipo v-cone para medição de vazão de gás natural em condições de gás úmido. Observa-se que os medidores do tipo placa de orifício, geralmente utilizados na medição de vazão de gás no segmento de Exploração e Produção (E\&P), podem não representar a tecnologia mais adequada. $O$ medidor v-cone representa uma tecnologia relativamente recente, que vem demonstrando bom desempenho para a medição de vazão e vem ganhando aceitação de órgãos reguladores. O interesse e a motivação do pesquisador residem na busca de maior exatidão na medição de vazão em condições operacionais adversas (gás úmido não especificado), sem impacto significativo sobre o custo do sistema. O presente trabalho, por meio de uma pesquisa bibliográfica e documental, apresenta o estágio atual de desenvolvimento da tecnologia de medição por v-cone e detalha as características dos sistemas de medição de vazão de gás natural, além de abordar as normas e regulamentos relacionados ao tema em questão. A fase experimental busca avaliar o desempenho do medidor por meio de uma investigação empírica realizada em laboratório, simulando condições de gás úmido encontradas em campo. Essa análise envolve também a determinação de erros e incertezas de medição. A pesquisa realizada, em conjunto com a avaliação dos resultados dos testes e das correlações utilizadas, demonstra que o medidor v-cone apresenta boa confiabilidade na medição de vazão de gás natural em escoamento de gás úmido, podendo ser utilizado em aplicações encontradas no segmento de E\&P.

\section{Palavras-chave}

Metrologia; medição de vazão; gás úmido; medidor tipo v-cone. 


\section{Abstract}

Barra Vidal de Oliveira, Thiago; Orlando, Alcir de Faro. Metrological evaluation of the v-cone type meter performance for wet gas flow rate measurement. Rio de Janeiro, 2010. 166p. MSc. Dissertation - Programa de Pós-Graduação em Metrologia. Departamento de Metrologia para Qualidade Industrial, Pontifícia Universidade Católica do Rio de Janeiro.

The aim of this study is to evaluate the metrological performance and potential of use of the v-cone type meter for measuring the natural gas flow rate under wet gas conditions. The orifice plate type meters, that are usually used to measure the gas flow rate in the Exploration and Production segment (E\&P), may not represent the most appropriate technology. The $v$-cone meter represents a relatively recent technology, which has shown good performance for the flow rate measurement and is gaining acceptance from the regulators. The interest and motivation of the researcher are related to the quest for greater flow rate measurement accuracy in adverse operating conditions (unspecified wet gas), with no significant impact on the cost of the system. This work, through a literature and documentary search, presents the current stage of development of the v-cone flow rate measurement technology and details the characteristics of natural gas flow rate measurement systems, in addition to addressing the standards and regulations relating to the subject. The experimental phase aims to evaluate the meter performance through an empirical research conducted in laboratory, by simulating wet gas conditions found in the field. This analysis also involves the determination of measurement errors and uncertainties. The research and the evaluation of test results and correlations show that the v-cone meter has good reliability in natural gas flow rate measurement under wet gas conditions, allowing it to be used in applications found in E\&P.

\section{Keywords}

Metrology; flow rate measurement; wet gas; v-cone type meter. 


\section{Sumário}

1 Introdução 15

1.1. Definição do problema de pesquisa 19

1.2. Objetivos geral e específicos 21

1.3. Metodologia 22

1.4. Motivação 23

1.5. Estrutura da dissertação 23

2 Fundamentação teórica $\quad 25$

2.1. Descrição geral do processamento primário de gás natural 25

2.2. Aproveitamento do gás natural 27

2.3. Conceitos 28

2.3.1. Número de Reynolds 29

$\begin{array}{ll}\text { 2.3.2. Calor específico } & 29\end{array}$

2.3.3. Coeficiente isentrópico ou coeficiente adiabático 29

2.3.4. Fator de compressibilidade 30

2.3.5. Lei da Continuidade ou Lei da Conservação da Massa 31

2.3.6. Lei da Conservação de Energia 31

2.3.7. Condensado de gás natural 32

2.3.8. Velocidade superficial 32

2.3.9. Parâmetro de Lockhart-Martinelli 33

2.3.10. Número de Froude $\quad 34$

2.3.11. Padrões de fluxo ou padrões de escoamento 34

2.4. Medição convencional de vazão de gás natural 37

2.5. Medição de vazão de gás natural em escoamento com presença de líquido $\quad 39$

2.5.1. Definição de gás úmido 40

2.6. Medidor do tipo v-cone ou cone invertido 41

2.6.1. Cálculo da vazão volumétrica de gás no medidor v-cone 48

2.6.1.1. Cálculo da vazão volumétrica em condições de referência 51

3 Metodologia para medição de vazão de gás em escoamento de gás úmido 52 
3.1. Correlações para correção da vazão de gás natural 54

3.1.1. Correlações desenvolvidas para placa de orifício 56

3.1.1.1. Correlação de Murdock $\quad 57$

3.1.1.2. Correlação de Chisholm 58

3.1.2. Correlações desenvolvidas para medidor venturi 59

3.1.2.1. Correlação de Leeuw 60

3.1.2.2. Correlação de Steven 61

3.1.3. Correlação desenvolvida para v-cone 63

3.1.3.1. Nova correlação de Steven 63

3.2. Circuito de testes para avaliação do v-cone 65

$\begin{array}{ll}\text { 3.3. Apresentação dos resultados dos testes } & 67\end{array}$

4 Resultados Experimentais $\quad 71$

4.1. Resultados da medição de vazão de gás do v-cone 72

4.1.1. Correção da medição de vazão de gás 78

4.1.1.1. Aplicação das correlações de Murdock e Chisholm 78

4.1.1.2. Aplicação das correlações de Leeuw e Steven 82

4.1.1.3. Aplicação da nova correlação de Steven 85

4.1.1.4. Correlação para v-cone com beta igual a 0,6 88

4.2. Avaliação do desempenho do medidor v-cone 91

4.2.1. Avaliação das incertezas de medição (metodologia 1) 91

4.2.2. Avaliação das incertezas de medição (metodologia 2) 99

4.3. Avaliação do desempenho do medidor v-cone considerando altas frações $\begin{array}{ll}\text { de líquido } & 101\end{array}$

4.3.1. Avaliação das incertezas de medição considerando altas frações de líquido 104

4.4. Determinação da vazão de líquido em um escoamento de gás úmido 111

4.4.1. Vazões de gás e líquido em um medidor v-cone 112

5 Conclusões e Recomendações 115

$\begin{array}{ll}\text { 5.1. Conclusões } & 115\end{array}$

$\begin{array}{ll}\text { 5.2. Recomendações } & 118\end{array}$

$\begin{array}{lr}6 \text { Referências bibliográficas } & 120\end{array}$ 
ANEXOS

ANEXO A - Portaria INMETRO/DIMEL no 166: Aprovação de modelo do medidor de vazão do tipo v-cone

ANEXO B - Dados obtidos nos testes realizados no laboratório NEL 


\section{Lista de figuras}

Figura 1 - Exemplos de pontos de medição fiscal compartilhada e medição $\begin{array}{ll}\text { de apropriação aplicados a três campos de produção } & 18\end{array}$

Figura 2 - Medidor de vazão de gás úmido (multifásico) 20

Figura 3 - Sistema típico de condicionamento de gás natural em uma $\begin{array}{ll}\text { Unidade de Produção } & 27\end{array}$

Figura 4 - Cadeia de movimentação do gás natural 28

Figura 5 - Exemplo de mapa de padrões de escoamento 36

Figura 6 - Medidor tipo v-cone $\quad 42$

Figura 7 - Sistema de medição típico com medidor v-cone 44

Figura 8 - Sistema de medição de vazão por placa de orifício 56

Figura 9 - Medidor venturi $\quad 59$

Figura 10 - Representação esquemática do circuito de testes do

$\begin{array}{ll}\text { laboratório NEL } & 67\end{array}$

Figura 11 - Exemplo de gráfico apresentando os erros na medição de vazão

$\begin{array}{ll}\text { de gás do v-cone } & 69\end{array}$

Figura 12 - Exemplo de gráfico apresentando o erro residual percentual na medição de vazão de gás do v-cone, após aplicação de $\begin{array}{ll}\text { determinada correlação } & 70\end{array}$

Figura 13 - Circuito de testes do laboratório NEL 72

Figura 14 - Dados obtidos de sobrestimação na medição de vazão de gás do v-cone 75

Figura 15 - Influência da pressão de operação na superestimação

$\begin{array}{ll}\text { de vazão de gás } & 76\end{array}$

Figura 16 - Influência da velocidade do gás na sobrestimação

da vazão obtida pelo v-cone 76

Figura 17 - Influência do número de Froude na medição de vazão

de gás ( $P=15$ bar)

Figura 18 - Influência do número de Froude na medição de vazão

de gás $(P=40$ bar $) \quad 78$

Figura 19 - Sobrestimação da vazão de gás e aplicação da

correlação de Murdock

Figura 20 - Erro residual da vazão de gás após a aplicação da 
correlação de Murdock

Figura 21 - Sobrestimação da vazão de gás e aplicação da

correlação de Chisholm

80

Figura 22 - Erro residual da vazão de gás após a aplicação da

correlação de Chisholm

Figura 23 - Sobrestimação da vazão de gás e aplicação da

correlação de Leeuw

82

Figura 24 - Erro residual da vazão de gás após a aplicação da

correlação de Leeuw

83

Figura 25 - Sobrestimação da vazão de gás e aplicação da correlação

de Steven desenvolvida para o venturi

Figura 26 - Erro residual da vazão de gás após a aplicação da correlação

de Steven desenvolvida para o venturi

Figura 27 - Sobrestimação da vazão de gás e aplicação da correlação de Steven desenvolvida com v-cone de beta igual a 0,55

Figura 28 - Erro residual da vazão de gás após a aplicação da correlação de Steven desenvolvida com v-cone de beta igual a 0,55

Figura 29 - Sobrestimação da vazão de gás e aplicação da correlação

de Steven desenvolvida com v-cone de beta igual a 0,75

Figura 30 - Erro residual da vazão de gás após a aplicação da correlação

de Steven desenvolvida com v-cone de beta igual a 0,75

87

Figura 31 - Sobrestimação da vazão de gás e aplicação do novo fator de correção (Eq. (50)) específico para v-cone de beta igual a 0,6

Figura 32 - Erro residual da vazão de gás após a aplicação do novo fator de correção (Eq. (50)) específico para v-cone de beta igual a 0,6

Figura 33 - Erro residual da vazão de gás após a aplicação da nova

correlação x Vazão de gás de referência

Figura 34 - Erro residual da vazão de gás após a aplicação da nova correlação x Parâmetro de Lockhart-Martinelli

Figura 35 - Vazão volumétrica de gás de referência $x$ Vazão volumétrica de gás corrigida

Figura 36 - Sobrestimação da vazão de gás e aplicação da nova correlação específica para v-cone de beta igual a 0,6, com $X$ variando entre 0 e 0,4

Figura 37 - Erro residual da vazão de gás após a aplicação da nova correlação específica para v-cone de beta igual a 0,6, 
com $X$ variando entre 0 e 0,4

Figura 38 - Erro residual da vazão de gás após a aplicação da nova correlação x Vazão de gás de referência, considerando $X$ até 0,4

Figura 39 - Erro residual da vazão de gás após a aplicação da nova correlação x Parâmetro de Lockhart-Martinelli $(X)$,

com $X$ variando entre 0 e 0,4

108

Figura 40 - Flutuação de pressão no v-cone 


\section{Lista de tabelas}

Tabela 1 - Laboratórios acreditados pela RBC para calibração volumétrica de medidores de vazão de gás natural

Tabela 2 - Análise comparativa das tecnologias de medição de vazão de gás natural

Tabela 3 - Coeficiente de Descarga $\left(C_{d}\right)$ obtido em cada condição operacional nos testes com gás seco

Tabela 4 - Erro máximo encontrado na vazão de gás natural 91

Tabela 5 - Erros médios e incertezas associadas a cada valor de erro 92

Tabela 6 - Incerteza associada a cada valor de vazão de gás 94

Tabela 7 - Erros médios e incertezas associadas a cada valor de erro 95

Tabela 8 - Incerteza associada a cada valor de parâmetro de

Lockhart-Martinelli

Tabela 9 - Incerteza associada a cada valor de vazão de gás obtida pela metodologia 2

Tabela 10 - Comparação das incertezas de medição obtidas pelas

metodologias 1 e 2

Tabela 11 - Erro máximo encontrado na vazão de gás natural, com $X$ variando entre 0 e 0,4

Tabela 12 - Erros médios e incertezas associadas a cada valor de erro

Tabela 13 - Incerteza associada a cada valor de vazão de gás

Tabela 14 - Erros médios e incertezas associadas a cada valor de erro

Tabela 15 - Incerteza associada a cada valor do parâmetro de

Lockhart-Martinelli

Tabela 16 - Dados do sistema de medição de vazão de gás de

referência - 15 bar

Tabela 17 - Dados do sistema de medição de vazão de líquido de

referência - 15 bar

Tabela 18 - Dados do sistema de medição de vazão com medidor

v-cone - 15 bar

Tabela 19 - Dados do sistema de medição de vazão de gás de referência - 20 bar

Tabela 20 - Dados do sistema de medição de vazão de líquido de 
referência - 20 bar

Tabela 21 - Dados do sistema de medição de vazão com medidor

v-cone - 20 bar

Tabela 22 - Dados do sistema de medição de vazão de gás de

referência - 30 bar

Tabela 23 - Dados do sistema de medição de vazão de líquido de referência - 30 bar

Tabela 24 - Dados do sistema de medição de vazão com medidor $\mathrm{v}$-cone - 30 bar

Tabela 25 - Dados do sistema de medição de vazão de gás de

referência - 40 bar

Tabela 26 - Dados do sistema de medição de vazão de líquido de referência - 40 bar

Tabela 27 - Dados do sistema de medição de vazão com medidor v-cone - 40 bar 